\title{
Fingerprint Ridge Distance Estimation: A Mathematical Modeling
}

\author{
Shing Chyi Chua \\ Multimedia University \\ Jalan Ayer Keroh Lama \\ 75450 Melaka, Malaysia
}

\author{
Eng Kiong Wong \\ Multimedia University \\ Jalan Ayer Keroh Lama \\ 75450 Melaka, Malaysia
}

\author{
Alan Wee Chiat Tan \\ Multimedia University \\ Jalan Ayer Keroh Lama \\ 75450 Melaka, Malaysia
}

\begin{abstract}
In this paper, fingerprint image is mathematically modeled by using a $2 \mathrm{D}$ sinusoidal function in a local window of size $32 \times 32$. The estimated ridge distance is then found by using the Levenberg-Marquardt gradient descent method. From test images, it has been found that the error percentage is $5 \%$ or less for fingerprint images of good to moderate quality with ridge distances between five and 20 pixels corrupted with zero mean white Gaussian noise of variance levels between zero and 1.
\end{abstract}

\section{General Terms}

Pattern Recognition, Gradient Descent

\section{Keywords}

Fingerprint ridge distance estimation

\section{INTRODUCTION}

Fingerprint ridge orientation estimation and ridge distance estimation remain two important steps in fingerprint image enhancement. Ridge distance refers to the average pixel distance between ridges. It can also be called ridge frequency obtained by computing the number of cycle per pixel. Finding the right ridges estimation algorithm for embedded environment is a vital part of automatic embedded fingerprint identification system (FIS) [1]. It is important to estimate the ridge distance for improving the performance of an automated FIS as ridge distance is one of the important parameter in fingerprint enhancement [2].

\begin{abstract}
Many literature have proposed ridge distance estimation and can broadly be categorized into two methods: spatial domain and spectral domain. Both methods use image blocks, the latter in addition, transforms the blocks into frequency domain for the ridge distance estimation. In [3] geometric approach and spectral approach in estimating the ridge frequency were introduced. In the geometric approach the central points of the ridges have been estimated on a regular grid and a ridge direction have been extracted. The distance between two ridges is found along the line normal to the ridge direction. The spectral approach uses the harmonic coefficients of the discrete Fourier transform (DFT) based on a radial distribution function. This is different from the conventional spectral approach using the peak spectral power in computing the ridge frequency such as in [4].
\end{abstract}

While in [1,5], statistical method has been used. The statistical method determines the ridge distance distribution in each block image through a histogram. It detects all possible peaks and calculates the intervals in the histogram as the ridge distance. Other similar methods that used peak intervals as well as valley intervals by utilizing the wavelike signature of the fingerprint in the estimation of the ridge distance within an oriented local window can be found in $[6,7,8]$. From the work in [9], mathematical characterization using 2D sinusoidal signal has been used. The calculation of local frequency uses the magnitude of all partial derivatives up to the order of two.

In this paper, fingerprint image is mathematically modeled by using a $2 \mathrm{D}$ sinusoidal function in a local window of size $32 \times 32$. The estimated ridge distance is then found by using the Levenberg-Marquardt gradient descent method (LMGD). The method described is easy to formulate and understand as gradient descent method has been widely used in many applications. The LMGD algorithm is a heuristic to improve the convergence time and it especially useful in nonlinear optimization problem such as this.

\section{METHODOLOGY}

Let the intensity image in the range between zero and one of a fingerprint image be denoted by $f(x, y)$. The image is partitioned into non-overlapping blocks of size $w \times x w$ (in this work $w=32$ ). Each of the blocks can mathematically be modeled as a 2D sinusoidal function given by

$f(x, y ; \omega, \theta, \phi)=0.5 \sin (a \omega+\phi)+0.5$

where $a=x \cos \theta+y \sin \theta, \omega$ is the spatial frequency of the sinusoidal grating, $\theta$ is the orientation of the normal to the grating, and $\phi$ is the amount of phase shift. Note that $\omega=2 \pi / T$ where $T$ is the ridge distance.

Let the image $I$ be a representation of a typical fingerprint image that has been corrupted by noise. Then the aim is to find estimations for $\omega, \theta$ and $\phi$ which minimize the square error given by

$e(\omega, \theta, \phi)=(f-I)^{2}$

To solve the problem, let the vector $\mathbf{X}=[\omega \cos \theta \omega \sin \theta \phi]^{\mathrm{T}}$. Hence, the vector representation of Eq. (1) is now given by

$f(A ; \mathbf{X})=0.5 \sin (A \mathbf{X})+0.5$

where $A=\left[\begin{array}{lll}x & y & 1\end{array}\right]$. It is well known that an estimation of the local orientation $\theta$ using block operation can be computed by using

$$
\left.\theta=\frac{1}{2} \tan ^{-1}\left(\sum_{u, v \in w \times w} 2 g_{x}(u, v) g_{y}(u, v) / \sum_{u, v \in w \times w} g_{x}^{2}(u, v)-g_{y}^{2}(u, v)\right)\right)
$$

where $u, v \in w \times w$ defines all the points in the 2D region of size $w \mathrm{x} w$ while $g_{x}$ and $g_{y}$ are the gradients in the directions $x$ and $y$, respectively $[6,10,11]$. As the parameter $\theta$ can independently be computed, it can be taken out from the vector $\mathbf{X}$ and Eq. (3) can be remodeled such that $\mathbf{X}=\left[\begin{array}{ll}\omega & \phi\end{array}\right]^{\mathrm{T}}$ and $A=$ $[x \cos \theta+y \sin \theta$ 1]. To solve for $\omega$ and $\phi$, LMGD method $[12,13]$ is used. The algorithm in general, is as follows:

1. Let an initial guess to the solution be $\mathbf{X}(0)=[\omega(0) \phi(0)]^{\mathrm{T}}$. 
Let an initial tuning parameter be $\lambda=\lambda(0)$.

2. For $j>0$, the subsequent $\mathbf{X}$ is found by using

$\mathbf{X}(j+1)=\mathbf{X}(j)-[H+\lambda \operatorname{diag}(H)]^{-1} \mathbf{D}$

where $H=\nabla f \nabla f^{T}$ represents the Hessian matrix and $\mathbf{D}=$ $(f-I) \nabla f$ is the derivative of $e . \lambda$ is an adjustable parameter according to the rules below.

3. If $e(\mathbf{X}(j+1))>e(\mathbf{X}(j))$, then $\mathbf{X}(j+1)=\mathbf{X}(j)$ and $\lambda=10 \lambda$. Else, $\lambda=\lambda / 10$.

4. Repeat step 2. Stop when $\mathbf{X}$ has converged.

The initial guess $\mathbf{X}(0)$ to be employed in the algorithm is crucial while the parameter $\lambda$ is actually a tunable step size for this convergence to occur. To make sure an appropriate initial guess is used, the space of $\omega$ and $\phi$ is first partitioned according to: 1) partition of $\omega=\{2 \pi / T \mid 5 \leq T \leq 20\}$ valid for $T=\{5,6,8,11,15,20\}$ and 2) partition of $\phi=\left\{0^{\circ}, 90^{\circ}, 180^{\circ}, 270^{\circ}\right\}$.

An estimation to $[\omega(0) \phi(0)]$ taken at discrete points of the partitions are found by solving Eq. (6).

$\operatorname{Min} e(\omega, \phi)=(f-I)^{2}$

Finally from the estimated value of $\omega$ after applying LMGD method, the estimated ridge distance (or the ridge period $T_{e}$ ) is as revealed in Eq. (7).

$T_{e}=2 \pi \omega($ in pixels)

Note that although by definitions, the ridge distance and ridge period are not the same [3]. In this paper, it is used interchangeably as is commonly found in most literatures.

\section{RESULTS AND DISCUSSION}

2D sinusoidal functions corresponding to locally windowed fingerprint images have been generated as test images and then corrupted by additive zero mean white Gaussian noise (ZWGN) of variance $\sigma^{2}$ as given by Eq. (8).

$I(x, y)=f(x, y)+\mathrm{ZWGN}\left(\sigma^{2}\right)$

where $\mathrm{ZWGN}\left(\sigma^{2}\right) \sim N\left(0, \sigma^{2}\right)$. The range of each variable used to generate the test images is:

i) range of $\omega=\{2 \pi|T| 5 \leq T \leq 20\}$ with step size of 0.5 ,

ii) range of $\theta=\left\{0^{\circ} \leq \theta<180^{\circ}\right\}$ with step size of $10^{\circ}$,

iii) range of $\phi=\left\{0^{\circ} \leq \phi<360^{\circ}\right\}$ with step size of $10^{\circ}$, and

iv) range of $\sigma^{2}=\left\{0 \leq \sigma^{2} \leq 1\right\}$ with step size of 0.1 .

Ridge distances of three to 20 pixels, and three to 25 pixels, respectively are stated to be reasonable values to be used in typical 500 dpi fingerprint images [3,6]. Adjacent ridges are expected to be located eight to 12 pixels apart [14] in 500dpi images and 10 pixels has been observed as the average ridge distance $[6,7,14,15,16]$. In this paper, the range $T$ used was $T_{\min }=5$ pixels and $T_{\max }=20$ pixels, which was based on half and doubling of the average ridge frequency $1 / T$ which was also used in [16]. This range stood comfortably within the common ridge distances mentioned.

For each ridge distance at different levels of noise variance, the percentage of error is calculated by averaging over all $\theta$ and $\phi$ given by Eq. (9).

\%error $=\frac{\left|T-<T_{e}>\right|}{T} \times 100 \%$
Fig. 1 shows the test images generated via ridge distance of 10 pixels, orientation of $0^{\circ}$, phase shift of $0^{\circ}$ and corrupted by different levels of noise variance.

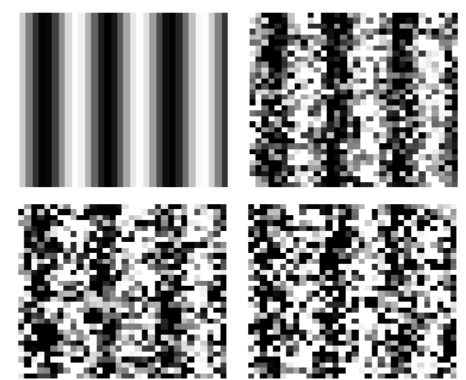

Fig. 1: Test images with noise levels $\sigma^{2}=0$ (top-left), $\sigma^{2}=0.2$ (top-right), $\sigma^{2}=0.5$ (bottom-left) and $\sigma^{2}=0.8$ (bottom-right).

Fig. 2 shows the graphs of error percentage corrupted by different levels of noise variance of $0,0.2,0.5$ and 0.8 . It also shows the error percentage of other commonly used methods (for comparison) as described in Table 1. The complete data of all ridge distances and noise levels are as attached in Table 2, Table 3, Table 4, and Table 5.

Table 1. Methods and descriptions

\begin{tabular}{|c|c|}
\hline Methods & Descriptions \\
\hline A & $\begin{array}{l}\text { A spectral analysis method using Fourier } \\
\text { transform (FT). The estimated ridge distance is } \\
\text { found by using } \\
\qquad T_{e}=1 / f, f=\sqrt{\left(u_{p k}-u_{o}\right)^{2}+\left(v_{p k}-v_{o}\right)^{2}} \\
\text { where }\left(u_{p k}, v_{p k}\right) \text { and }\left(u_{o}, v_{o}\right) \text { are the coordinate } \\
\text { points corresponding to the peak FT spectral } \\
\text { and the DC value, respectively. For example, } \\
\text { similar work can be found in }[3,4] \text {. }\end{array}$ \\
\hline B & $\begin{array}{l}\text { A spectral analysis method using FT. The } \\
\text { estimated ridge distance is found by using radial } \\
\text { distribution according to } \\
T_{e}=\frac{1}{\arg \{Q(r)\}}, Q(r)=\frac{1}{\# C_{r}} \sum_{(u, v) \in C_{r}}|G(u, v)| \\
\text { max } \\
\text { of } \# C_{r} \text { is the number of elements in the set } \\
r \approx \sqrt{\left(u-u_{o}\right)^{2}+\left(v-v_{o}\right)^{2}} \text { and } G(u, v) \text { is the } \\
\text { FT. For example, similar work can be found in } \\
{[3,5] .}\end{array}$ \\
\hline $\mathrm{C}$ & $\begin{array}{l}\text { A spatial domain method. The ridge lines are } \\
\text { either projected along its orientation direction or } \\
\text { its grey-levels profile is found in the direction } \\
\text { orthogonal to the orientation [1,3,5,6,7]. This } \\
\text { method is used due to the periodic nature of the } \\
\text { ridges. The estimated ridge period may be found } \\
\text { by using } T_{e}=\frac{1}{N} \sum_{i=1}^{N}\left(R_{i}+V_{i}\right) \text { where } R_{i} \text { and } V_{i} \\
\text { are the ridge width and valley width [3]. }\end{array}$ \\
\hline
\end{tabular}




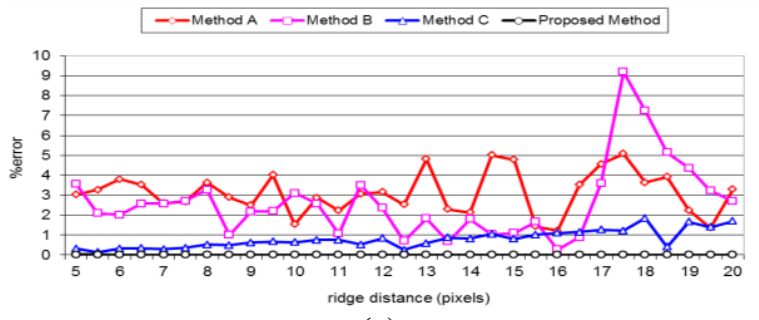

(a)

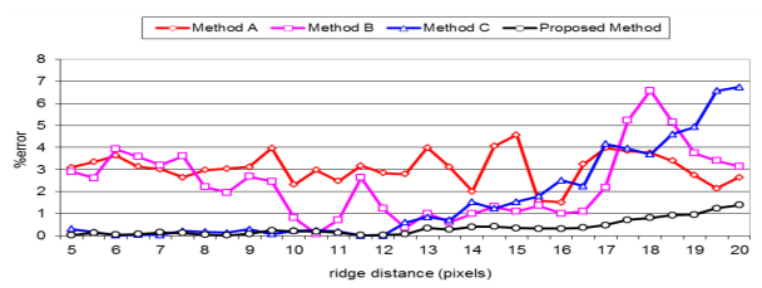

(b)

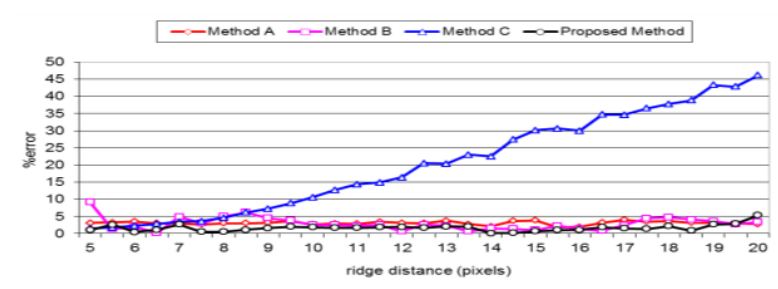

(c)

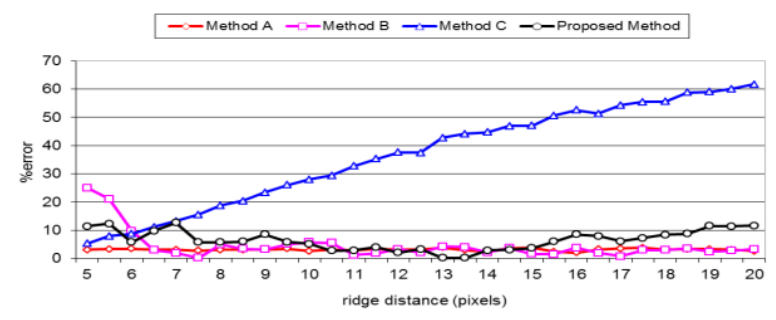

(d)

Fig. 2: Percentage of error for ridge distances 5 to 20 with noise variance a) $\sigma^{2}=0$, b) $\sigma^{2}=0.2$, c) $\sigma^{2}=0.5$, d) $\sigma^{2}=0.8$

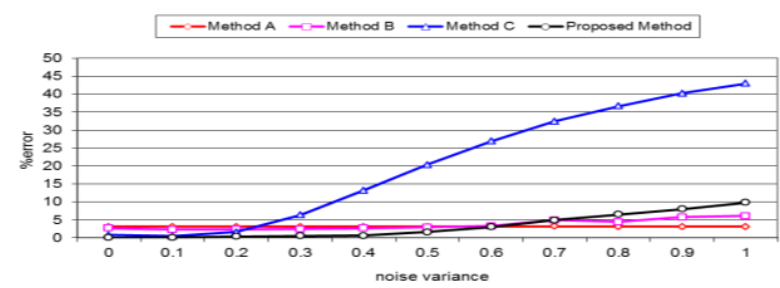

Fig. 3: Overall percentage error based on noise variance for ridge distance 5 to 20 pixel
It has been observed that the proposed method consistently scored at percentage of error of at most $5 \%$ which is lower as compared to other methods as shown in Fig. 2(a-c) with the exception when the images are highly corrupted (noise variance 0.7 and above) in Fig. 2d. The overall error percentage averaged over all the ridge distances is as illustrated in Fig. 3 and shows low error (5\%) below noise variance of around $\sigma^{2}=0.7$. Shorter and larger ridge distances might not occurred as frequently as the average ridge distance is about 10 pixels, and thus the error percentage might be much lower than observed. Adjacent ridges are expected to be located eight to 12 pixels apart in 500dpi images with an average of 10 pixels [14]. The proposed method is consistently better than Methods A, B and C unless the noise variance gets too high ( 0.7 and above). With the assumption that majority fingerprint images are of good to moderate qualities, the proposed method would on average be the best in keeping the error in ridge distance low.

The time complexity graph is as shown in Fig. 4. The average time consumed is $6.9 \mathrm{~ms}, 22.4 \mathrm{~ms}, 6.7 \mathrm{~ms}$ and $8.1 \mathrm{~ms}$, respectively. The proposed method shows increment in time complexity as noise variance increases. Method A is consistent in its time complexity while although Method C is the faster, the error percentage increases almost linearly beyond a certain noise variance. This is due to the difficulty to reliably detect consecutive peaks of gray-levels in noisy fingerprint images using the spatial projection method [17].

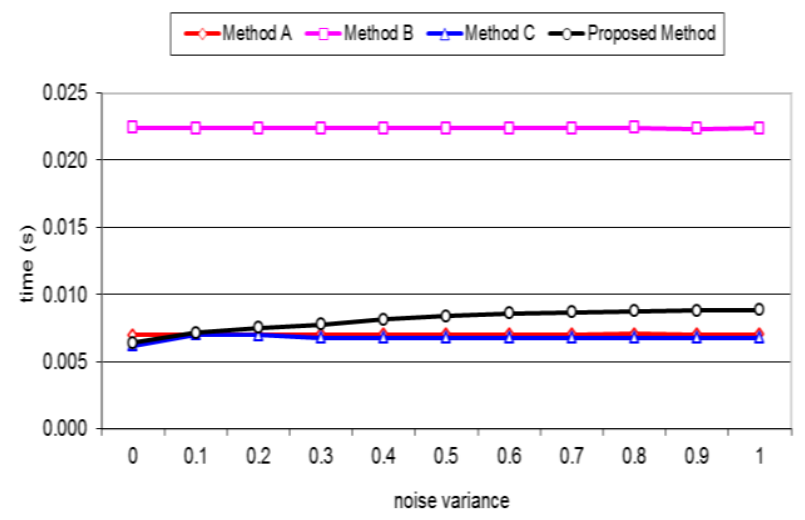

Fig. 4: Time complexity 

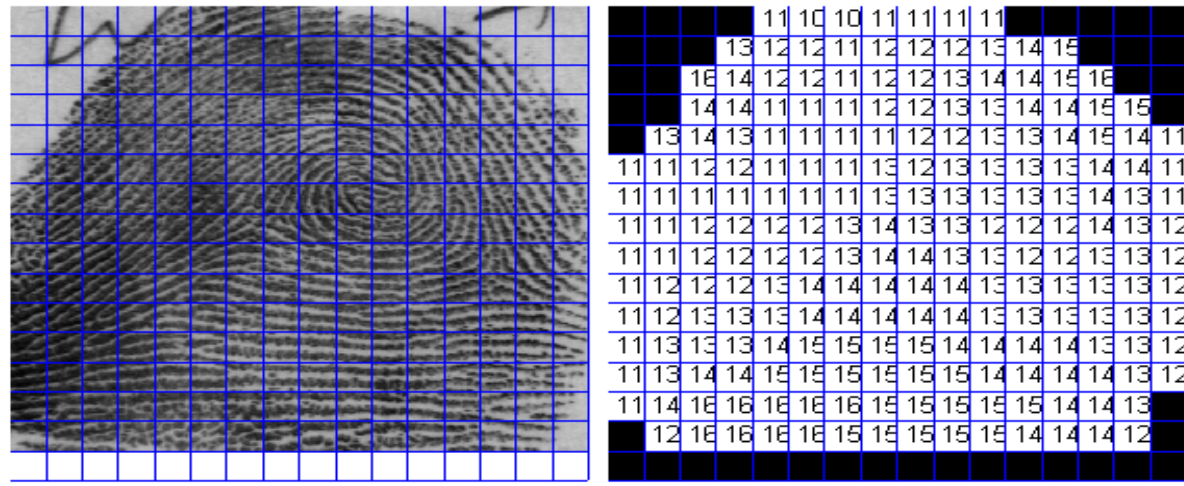

Fig. 5: Fingerprint F0001_01 NIST-SD4
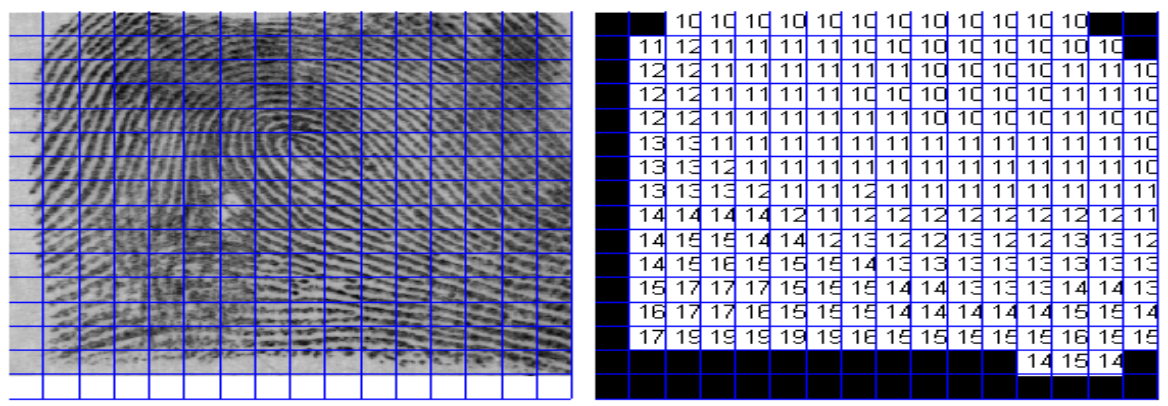

Fig. 6: Fingerprint F0002_05 NIST-SD4

Fig. 5 and Fig. 6 show the results on two real fingerprint images taken from NIST special database 4 (NIST-SD4) [18]. In real fingerprint images, where the quality of the blocks may vary, interpolation and lowpass filtering may be used to estimate the ridge distance over a neighborhood of the corrupted regions [6]. The final estimated ridge distances depicted have been median filtered about its neighborhood and then rounded for display. Segmentation has also been done to omit background regions. All simulations are carried in Matlab using 32-bit, 2038MB RAM, Duo $1.5 \mathrm{GHz}$ Intel Core Windows Vista.

\section{CONCLUSION}

The authors have applied mathematical modeling for the estimation of ridge distance using test images that represent local characteristic of fingerprints using LMGD method. The performance is evaluated with the test images corrupted with ZWGN over noise variance between 0 and 1 using the percentage error as the indicator. The proposed method has on average $5 \%$ or less in percentage of error for good and moderate quality images over a range of ridge distances between five and 20 pixels, suitable for typical for fingerprint images at $500 \mathrm{dpi}$ of resolution with average ridge distance of 10 pixels.

\section{REFERENCES}

[1] Wang, F., Yang, D., Cao, H., Sun, G., and Hu, Y., The Improved Fingerprint Ridge Distance Extraction Algorithm Based on Embedded Environment, Journal of Information \& Computational Science 9(14) (2012), 4187-4197.

[2] Zhan, X., Sun, Z., Yin, Y., and Chu, Y., Fingerprint Ridge Distance Estimation: Algorithms and the Performance, Advances in Biometrics LNCS 3832 (2005), 294-301.
[3] Kovacs-Vajna, Z.M., Rovatti, R., and Frazzoni, M., Fingerprint Ridge Distance Computation Methodologies, Pattern Recognition 33 (2000), 69-80.

[4] Sutthiwichaiporn, P., and Areekul, V., Adaptive Boosted Spectral Filtering for Progressive Fingerprint Enhancement, Pattern Recognition 46 (2013), 2465 2486.

[5] Yin, Y., Tian, J., and Yang, X., Ridge Distance Estimation in Fingerprint Images: Algorithm and Performance Evaluation, EURASIP Journal on Applied Signal Processing (2004), 495-502.

[6] Hong, L., Wan, Y., and Jain, A., Fingerprint Image Enhancement: Algorithm and performance Evaluation, IEEE Transactions on Pattern Analysis and machine intelligence 20(8) (1998), 777-789.

[7] Porwik, P., and Wieclaw, L., A New Fingerprint Ridges Frequency Determination Method. IEICE Electronic Express 6(3) (2009), 154-160.

[8] Babatunde, I.G. , Charles, A.O., and Olatunbosun, O., Uniformity Level Approach to Fingerprint Ridge Frequency Estimation, International Journal of Computer Application 61(22) (2013), 26-32.

[9] Maio, D., and Maltoni, D., Ridge-line Density Estimation in Digital Images, 14th International Conference on Pattern Recognition 1 (1998), 534-538.

[10] Kass, M., and Witkin, A., Analyzing Oriented Pattern, Computer Vision, Graphic, and Image Processing 37(3) (1987), 362-385.

[11] Rao, A., A Taxonomy for Texture Description and Identification, Springer-Verlag, New York, 1990.

[12] Marquardt, D.W., An Algorithm for Least-squares Estimation of Nonlinear Parameters, Journal of the 
Society for Industrial and Applied Mathematics 11(2) (1963), 431-441.

[13] Roweis, S., Levenberg-Marquardt Optimization, Available online: http://www.cs.nyu.edu/ roweis/notes/lm.pdf, last accessed: Apr. 2015.

[14] Bazen, A.M., and Gerez, S.H., Systematic Methods for the Computation of the Directional Fields and Singular Points of Fingerprints, IEEE Transactions on Pattern Analysis and Machine Intelligence 24(7) (2002), 905919.

[15] Jain, A.K., Prabhakar, S., Hong, L., and Pankanti, S., Filterbank-based Fingerprint Matching, IEEE Transactions on Image Processing 9(5) (2000), 846-859.
[16] Hastings, R., Ridge Enhancement in Fingerprint Images Using Oriented Diffusion, 9th Biennial Conference of the Australian Pattern Recognition Society on Digital Image Computing Techniques and Applications (2007), 245252.

[17] Maltoni, D., Maio, D., Jain, A.K., and Prabhakar, S., Handbook of Fingerprint Recognition, Springer, 2009.

[18] Watson, C.I. and Wilson, C.L., NIST Special Database 4, Fingerprint Database, National Institute of Standard and Technology, 1992.

\section{APPENDIX}

Table 2. Error percentage of ridge distance corrupted by different noise variance for Method A

\begin{tabular}{|c|c|c|c|c|c|c|c|c|c|c|c|}
\hline & \multicolumn{10}{|c|}{ Noise variance } \\
\hline Ridge distance & $\mathbf{0}$ & $\mathbf{0 . 1}$ & $\mathbf{0 . 2}$ & $\mathbf{0 . 3}$ & $\mathbf{0 . 4}$ & $\mathbf{0 . 5}$ & $\mathbf{0 . 6}$ & $\mathbf{0 . 7}$ & $\mathbf{0 . 8}$ & $\mathbf{0 . 9}$ & $\mathbf{1}$ \\
\hline 5 & 3.02 & 3.04 & 3.09 & 3.05 & 3.08 & 3.07 & 3.12 & 3.10 & 3.04 & 3.07 & 3.14 \\
\hline 6 & 3.81 & 3.70 & 3.64 & 3.61 & 3.55 & 3.49 & 3.47 & 3.36 & 3.46 & 3.43 & 3.24 \\
\hline 7 & 2.59 & 2.92 & 3.01 & 3.09 & 3.12 & 3.03 & 3.03 & 3.03 & 3.08 & 3.05 & 3.21 \\
\hline 8 & 3.64 & 3.14 & 2.98 & 3.14 & 2.94 & 2.99 & 3.01 & 2.86 & 3.07 & 2.80 & 2.98 \\
\hline 9 & 2.49 & 3.03 & 3.13 & 3.19 & 3.11 & 3.18 & 3.36 & 3.01 & 3.16 & 3.38 & 3.37 \\
\hline 10 & 1.53 & 1.98 & 2.31 & 2.48 & 2.51 & 2.72 & 2.61 & 2.93 & 2.65 & 2.70 & 2.74 \\
\hline 11 & 2.23 & 2.33 & 2.48 & 2.50 & 2.65 & 2.81 & 2.91 & 2.91 & 2.78 & 2.83 & 2.99 \\
\hline 12 & 3.15 & 2.67 & 2.84 & 2.77 & 3.00 & 3.06 & 3.11 & 3.07 & 3.13 & 3.10 & 3.14 \\
\hline 13 & 4.81 & 4.28 & 3.99 & 3.81 & 3.78 & 3.77 & 3.63 & 3.84 & 3.83 & 3.62 & 3.22 \\
\hline 14 & 2.12 & 2.08 & 2.01 & 2.13 & 2.07 & 2.11 & 2.19 & 2.39 & 2.52 & 2.63 & 2.58 \\
\hline 15 & 4.79 & 4.78 & 4.57 & 4.44 & 4.06 & 3.81 & 3.78 & 3.79 & 3.79 & 3.80 & 3.58 \\
\hline 16 & 1.22 & 1.38 & 1.51 & 1.68 & 2.02 & 1.96 & 2.27 & 2.09 & 2.07 & 2.45 & 2.09 \\
\hline 17 & 4.56 & 4.06 & 3.98 & 3.94 & 3.74 & 3.97 & 3.62 & 3.96 & 3.55 & 3.82 & 3.66 \\
\hline 18 & 3.64 & 3.58 & 3.76 & 3.70 & 3.52 & 3.64 & 3.35 & 3.36 & 3.07 & 3.44 & 3.36 \\
\hline 19 & 2.24 & 2.65 & 2.73 & 2.84 & 2.90 & 3.07 & 3.16 & 3.17 & 3.25 & 3.27 & 3.28 \\
\hline 20 & 3.28 & 2.78 & 2.65 & 2.63 & 3.06 & 2.78 & 2.94 & 2.81 & 2.63 & 3.04 & 2.66 \\
\hline
\end{tabular}

Table 3. Error percentage of ridge distance corrupted by different noise variance for Method B

\begin{tabular}{|c|c|c|c|c|c|c|c|c|c|c|c|}
\hline \multirow[b]{2}{*}{ Ridge distance } & \multicolumn{11}{|c|}{ Noise variance } \\
\hline & $\mathbf{0}$ & 0.1 & 0.2 & 0.3 & 0.4 & 0.5 & 0.6 & 0.7 & 0.8 & 0.9 & 1 \\
\hline 5 & 3.56 & 3.62 & 2.91 & 2.77 & 1.57 & 9.12 & 11.91 & 26.76 & 24.98 & 54.33 & 38.95 \\
\hline 6 & 2.00 & 2.73 & 3.92 & 4.72 & 5.28 & 1.92 & 0.82 & 6.25 & 9.74 & 7.65 & 20.24 \\
\hline 7 & 2.57 & 2.37 & 3.20 & 3.76 & 6.00 & 4.90 & 0.63 & 2.19 & 1.87 & 0.46 & 1.77 \\
\hline 8 & 3.27 & 3.01 & 2.22 & 4.76 & 5.09 & 5.01 & 5.18 & 5.33 & 4.86 & 5.93 & 4.13 \\
\hline 9 & 2.18 & 2.13 & 2.68 & 3.86 & 5.74 & 4.51 & 6.62 & 7.80 & 3.21 & 7.77 & 3.57 \\
\hline 10 & 3.10 & 1.18 & 0.82 & 1.93 & 2.38 & 2.50 & 4.33 & 3.65 & 5.69 & 6.66 & 2.52 \\
\hline 11 & 1.07 & 0.87 & 0.72 & 0.35 & 0.98 & 2.25 & 3.66 & 1.11 & 1.24 & 3.95 & 3.17 \\
\hline 12 & 2.36 & 1.95 & 1.24 & 1.19 & 0.41 & 0.71 & 1.74 & 3.61 & 3.28 & 6.31 & 6.50 \\
\hline 13 & 1.84 & 0.84 & 1.00 & 1.16 & 1.73 & 2.26 & 3.26 & 4.64 & 4.23 & 3.58 & 4.94 \\
\hline 14 & 1.80 & 1.31 & 1.00 & 0.97 & 1.59 & 1.55 & 1.64 & 2.75 & 2.11 & 1.57 & 2.62 \\
\hline 15 & 1.11 & 0.54 & 1.10 & 0.13 & 1.23 & 1.03 & 2.19 & 0.78 & 1.61 & 1.80 & 3.05 \\
\hline 16 & 0.26 & 0.35 & 1.01 & 2.72 & 1.83 & 1.38 & 1.72 & 1.75 & 3.70 & 0.18 & 0.92 \\
\hline 17 & 3.61 & 3.13 & 2.20 & 3.07 & 2.83 & 2.44 & 2.63 & 3.98 & 0.78 & 1.34 & 0.83 \\
\hline 18 & 7.23 & 7.07 & 6.58 & 6.06 & 5.38 & 4.76 & 3.56 & 4.24 & 2.94 & 2.62 & 1.04 \\
\hline
\end{tabular}




\begin{tabular}{|l|l|l|l|l|l|l|l|l|l|l|l|}
\hline 19 & 4.36 & 3.82 & 3.74 & 3.54 & 3.33 & 3.61 & 3.42 & 3.39 & 2.37 & 2.60 & 1.43 \\
\hline 20 & 2.72 & 2.80 & 3.15 & 3.23 & 3.88 & 3.35 & 3.79 & 3.72 & 3.25 & 3.20 & 2.88 \\
\hline
\end{tabular}

Table 4. Error percentage of ridge distance corrupted by different noise variance for Method C

\begin{tabular}{|c|c|c|c|c|c|c|c|c|c|c|c|}
\hline & \multicolumn{11}{|c|}{ Noise variance } \\
\hline Ridge distance & $\mathbf{0}$ & 0.1 & 0.2 & 0.3 & 0.4 & 0.5 & 0.6 & 0.7 & 0.8 & 0.9 & 1 \\
\hline 5 & 0.32 & 0.21 & 0.31 & 0.50 & 1.02 & 1.52 & 2.55 & 4.13 & 5.24 & 5.07 & 7.23 \\
\hline 6 & 0.32 & 0.09 & 0.02 & 0.24 & 0.99 & 2.23 & 3.73 & 6.41 & 8.76 & 11.60 & 13.45 \\
\hline 7 & 0.28 & 0.11 & 0.03 & 0.24 & 1.55 & 3.44 & 7.01 & 10.55 & 13.18 & 15.50 & 18.36 \\
\hline 8 & 0.51 & 0.27 & 0.17 & 0.50 & 2.33 & 4.59 & 9.76 & 13.86 & 18.72 & 21.51 & 24.36 \\
\hline 9 & 0.62 & 0.14 & 0.30 & 0.86 & 3.58 & 7.22 & 13.91 & 18.92 & 23.41 & 26.73 & 29.91 \\
\hline 10 & 0.63 & 0.51 & 0.23 & 1.50 & 5.10 & 10.55 & 16.17 & 22.98 & 28.01 & 32.03 & 34.15 \\
\hline 11 & 0.76 & 0.58 & 0.19 & 1.96 & 7.06 & 14.35 & 20.74 & 27.56 & 32.76 & 34.65 & 41.35 \\
\hline 12 & 0.83 & 0.54 & 0.00 & 3.11 & 8.88 & 16.40 & 23.62 & 32.10 & 37.62 & 40.86 & 44.87 \\
\hline 13 & 0.59 & 0.56 & 0.85 & 5.17 & 12.63 & 20.38 & 29.75 & 36.46 & 42.77 & 45.78 & 49.77 \\
\hline 14 & 0.82 & 0.79 & 1.52 & 5.79 & 14.47 & 22.47 & 33.82 & 39.12 & 44.74 & 48.94 & 52.61 \\
\hline 15 & 0.80 & 0.67 & 1.54 & 8.06 & 18.08 & 30.09 & 39.16 & 43.96 & 47.09 & 53.28 & 54.94 \\
\hline 16 & 1.11 & 0.46 & 2.51 & 10.91 & 21.88 & 29.99 & 39.66 & 47.42 & 52.57 & 56.37 & 56.99 \\
\hline 17 & 1.27 & 0.66 & 4.16 & 13.72 & 25.18 & 34.64 & 45.18 & 49.30 & 54.29 & 58.83 & 61.26 \\
\hline 18 & 1.85 & 0.75 & 3.69 & 15.97 & 26.16 & 37.73 & 45.87 & 52.43 & 55.57 & 61.52 & 61.85 \\
\hline 19 & 1.66 & 0.14 & 4.93 & 17.94 & 31.03 & 43.31 & 50.22 & 55.42 & 59.07 & 62.48 & 64.15 \\
\hline 20 & 1.70 & 0.16 & 6.73 & 19.91 & 36.04 & 46.11 & 52.70 & 57.93 & 61.76 & 61.59 & 64.84 \\
\hline
\end{tabular}

Table 5. Error percentage of ridge distance corrupted by different noise variance for the Proposed Method

\begin{tabular}{|c|c|c|c|c|c|c|c|c|c|c|c|}
\hline \multirow[b]{2}{*}{ Ridge distance } & \multicolumn{11}{|c|}{ Noise variance } \\
\hline & $\mathbf{0}$ & 0.1 & 0.2 & 0.3 & 0.4 & 0.5 & 0.6 & 0.7 & 0.8 & 0.9 & 1 \\
\hline 5 & 0.02 & 0.02 & 0.02 & 0.03 & 0.06 & 0.99 & 2.70 & 8.65 & 11.40 & 16.07 & 24.95 \\
\hline 6 & 0.01 & 0.02 & 0.04 & 0.01 & 0.04 & 0.48 & 1.59 & 3.72 & 5.74 & 10.65 & 14.78 \\
\hline 7 & 0.01 & 0.06 & 0.16 & 0.28 & 0.74 & 2.67 & 5.84 & 8.39 & 12.62 & 11.46 & 16.97 \\
\hline 8 & 0.00 & 0.02 & 0.05 & 0.13 & 0.07 & 0.51 & 1.61 & 3.64 & 5.66 & 6.23 & 8.86 \\
\hline 9 & 0.00 & 0.06 & 0.08 & 0.10 & 0.01 & 1.57 & 2.95 & 3.92 & 8.45 & 7.49 & 8.16 \\
\hline 10 & 0.00 & 0.09 & 0.21 & 0.35 & 0.48 & 1.84 & 1.26 & 2.68 & 5.09 & 5.18 & 6.25 \\
\hline 11 & 0.00 & 0.03 & 0.14 & 0.33 & 0.74 & 1.70 & 1.41 & 1.82 & 2.79 & 3.45 & 3.85 \\
\hline 12 & 0.00 & 0.01 & 0.03 & 0.27 & 0.55 & 1.94 & 2.85 & 2.49 & 2.03 & 2.67 & 2.61 \\
\hline 13 & 0.00 & 0.13 & 0.35 & 0.86 & 1.49 & 2.10 & 1.36 & 1.84 & 0.22 & 0.12 & 1.59 \\
\hline 14 & 0.00 & 0.11 & 0.40 & 0.62 & 1.46 & 0.10 & 0.60 & 0.92 & 2.81 & 2.42 & 4.65 \\
\hline 15 & 0.00 & 0.04 & 0.35 & 0.76 & 0.68 & 0.67 & 2.61 & 2.63 & 3.59 & 6.16 & 6.82 \\
\hline 16 & 0.00 & 0.05 & 0.33 & 0.59 & 0.44 & 1.10 & 2.59 & 5.30 & 8.52 & 8.61 & 10.16 \\
\hline 17 & 0.00 & 0.08 & 0.48 & 0.93 & 0.58 & 1.49 & 3.33 & 6.97 & 6.07 & 9.94 & 11.81 \\
\hline 18 & 0.00 & 0.25 & 0.81 & 1.27 & 0.89 & 2.16 & 4.59 & 7.36 & 8.38 & 11.07 & 12.40 \\
\hline 19 & 0.00 & 0.26 & 0.95 & 0.85 & 0.19 & 2.65 & 4.58 & 7.17 & 11.50 & 13.86 & 15.11 \\
\hline 20 & 0.00 & 0.22 & 1.40 & 0.65 & 0.82 & 5.34 & 6.87 & 9.79 & 11.65 & 14.03 & 14.83 \\
\hline
\end{tabular}

